TRI-PP-01-08

\title{
Charmed baryons in lattice QCD
}

\author{
Randy Lewis ${ }^{1}$, N. Mathur ${ }^{2}$ and R.M. Woloshyn ${ }^{2}$ \\ ${ }^{1}$ Department of Physics, University of Regina, Regina, SK, Canada S4S 0A2 \\ ${ }^{2}$ TRIUMF, 4004 Wesbrook Mall, Vancouver BC, Canada V6T 2 A3
}

\begin{abstract}
Masses of singly and doubly charmed baryons are calculated in quenched lattice QCD using an improved action of the D234 type on an anisotropic lattice. The mass differences between spin $3 / 2$ and spin $1 / 2$ baryon states are calculated and compared to mass differences between vector and pseudoscalar mesons. The suppression of spin splittings in mesons containing heavy quarks, characteristic of quenched QCD simulations, is not observed in the baryon sector. The mass dependence of color hyperfine effects is discussed within the context of the quark model and heavy quark effective theory.
\end{abstract}




\section{INTRODUCTION}

Quenched lattice quantum chromodynamics (QCD) does reasonably well at describing hadronic phenomena. However there are a few well established instances where quenched approximation clearly fails to reproduce experimental values. One well studied example is the persistent underestimate of mass differences between vector and pseudoscalar mesons containing heavy quarks [1]. While the situation is still unsettled, the first attempts with unquenched simulations do not yet resolve this discrepancy [2 [4] so, clearly, a better understanding of color hyperfine effects in lattice QCD would be helpful. One way to proceed is to explore the situation in heavy baryons. Unfortunately, this is not so easy since spin splittings in the baryon sector are smaller than in mesons while lattice QCD correlators for baryons (especially spin 3/2 states) are noisier than those of mesons. An early work [5] suggested that spin splittings in heavy baryons are indeed very small, but later studies for charmed baryons [6] and for bottom baryons [7] obtained results more in line with phenomenological expectations.

In this paper we present further results for charmed baryons using the approach of [6]. Calculations were carried out for lattices with different values of the lattice spacing. As well, the physical size of the lattice was increased compared to that used in [6].

Section II presents some details of the lattice simulation. The calculations are done with an improved action of the D234 type [8] on an anisotropic lattice. This action has been used and described previously so the detailed expressions are relegated to an Appendix. The results are given in Section III along with some discussion about the fitting procedure and about the estimate of systematic errors. No attempt was made in the present work to do a continuum extrapolation. At each lattice spacing, a number of systematic effects have been identified and estimates were made for uncertainties induced in masses and mass differences. These include the overall scale uncertainty, the choice of time window for fitting correlation functions, ambiguity in fixing the strange and charm quark mass and in the extrapolation to physical up and down quark masses.

In section IV we try to compare spin splittings in baryons with those in mesons. It turns out that, for a subset of mesonic and baryonic states, there is a very simple mass de-

pendence of color hyperfine effects. In fact over the whole mass range where experimental masses are available the ratio of meson to baryon mass difference is remarkably constant. This fact is used as a benchmark against which to view our lattice QCD results. It is suggested that, whereas quenched lattice QCD underestimates mesonic spin splittings, in baryons the situation is qualitatively different with no suppression of spin splittings being observed. Rather there is a tendency for spin splittings to be overestimated.

\section{METHOD}

The calculation is done on an anisotropic lattice using gauge field and quark actions that are improved [8] by removing tree level errors up to $O\left(a^{2}\right)$ and introducing renormalization factors for the gauge links to remove the dominant errors due to tadpole 
fluctuations. The tadpole factors are estimated using the expectation value of the gauge field link in Landau gauge. These actions have been used previously for heavy-light mesons [9] and in a preliminary study of charmed baryons [6]. The expressions for the actions are given in the appendix.

Hadron masses are calculated from zero-momentum correlation functions in the usual way. This choice of interpolating operators for the hadrons is not unique. For $\Sigma$-like baryons containing quarks with two different flavors (denoted by Q and q) a common choice $[10]$ is

$$
\epsilon^{a b c}\left[q_{a}^{T} C \gamma_{5} Q_{b}\right] q_{c}
$$

where a,b,c are color indices and Dirac indices have been suppressed. Taking $q=u$ and $Q=d$, this operator will give the usual interpolating operator for the proton and, as discussed in Ref. [6], it is advantageous to use this operator for all flavor combinations as this gives a unified analysis over the whole mass range. For the $\Xi_{Q}^{\prime}$ which has light quarks of different flavor coupled to spin 1, the operator

$$
\frac{1}{\sqrt{2}}\left\{\epsilon^{a b c}\left[q_{a}^{\prime T} C \gamma_{5} Q_{b}\right] q_{c}+\epsilon^{a b c}\left[q_{a}^{T} C \gamma_{5} Q_{b}\right] q_{c}^{\prime}\right\}
$$

symmetrized in the light quarks is used. The $\Lambda$-like baryons containing a heavy quark can be interpolated using an operator of the form

$$
\epsilon^{a b c}\left[q_{a}^{T} C \gamma_{5} q_{b}^{\prime}\right] Q_{c}
$$

A more symmetrical choice would be the octet lambda

$$
\frac{1}{\sqrt{6}} \epsilon^{a b c}\left\{2\left[q_{a}^{T} C \gamma_{5} q_{b}^{\prime}\right] Q_{c}+\left[q_{a}^{T} C \gamma_{5} Q_{b}\right] q_{c}^{\prime}-\left[q_{a}^{\prime T} C \gamma_{5} Q_{b}\right] q_{c}\right\}
$$

which is degenerate with the $\Sigma$ in the $\mathrm{SU}(3)$ flavor limit. This choice is consistent with the idea of using operators that allow for a unified calculation over all masses. In fact, it was found that the operators (2.3) and (2.4) give masses which are consistent within statistical errors so results using (2.4) are reported in this paper.

For spin $3 / 2$ states we use the simple operator (appropriate for the heavy quark limit)

$$
\epsilon^{a b c}\left[q_{a}^{T} C \gamma_{\mu} q_{b}^{\prime}\right] Q_{c}
$$

for all states since there are indications [11] that the more symmetrical form used in [6] leads to essentially identical results.

The spin $3 / 2$ field (2.5) propagates both spin $1 / 2$ and spin $3 / 2$ states [12]. At zero momentum the correlation function with spatial Lorentz indices has the general form [5]

$$
C_{i j}(t)=\left(\delta_{i j}-\frac{1}{3} \gamma_{i} \gamma_{j}\right) C_{3 / 2}(t)+\frac{1}{3} \gamma_{i} \gamma_{j} C_{1 / 2}(t)
$$


where the subscripts $3 / 2$ and $1 / 2$ denoted the spin projections. By choosing different Lorentz components the quantity $C_{3 / 2}(t)$ is extracted and used to determine the mass of the spin $3 / 2$ states.

Hadron correlators were calculated using interpolating operators in local form at both source and sink and also applying a gauge invariant smearing to the quark propagators at the sink. The gauge invariant smearing function, Eq. (13) of Ref. [13], was used. Hadron masses were obtained by a simultaneous fit to local and sink-smeared correlators taking into account correlations between different time slices with the inverse of the covariance matrix obtained using singular value decomposition. Two exponential functions are used in the fit to the local correlator. The sink-smeared correlation function is fit by a single exponential using a mass parameter constrained to be the same as the ground state mass of the fit to the local correlator. The time window for the fit was obtained by fixing the ending time to be sufficiently large so that the fits were not sensitive to its value and by varying the starting time so that a minimum of $\chi^{2}$ (typically around 1/d.o.f.) was achieved. Fits with starting times \pm 1 time step away from the time giving the minimum

$\chi^{2}$ were used to estimate the systematic uncertainty associated with fixing the fitting time window.

\section{RESULTS}

Calculations were carried out for three different sets of quenched gauge configurations on anisotropic lattices with a bare aspect ratio $a_{s} / a_{t}=2$ and spatial lattice spacing varying from about $0.22 \mathrm{fm}$ to $0.15 \mathrm{fm}$. Gauge fields were constructed using pseudo-heatbath Monte Carlo with $400(\beta=2.1)$ to $800(\beta=2.5)$ sweeps between saved configurations. Fixed time boundaries were used in calculating the quark propagators. The parameters for the lattices are given in Table $\mathbb{\mathbb { L }}$.

Six values of the hopping parameter were used and are listed in Table III. The smallest values were chosen in the region of the charm quark and the four larger values were used for extrapolating to the light $(\mathrm{u}, \mathrm{d})$ quark mass. The temporal lattice spacing was fixed by calculating the $\rho$-meson mass. As well known, quenched lattice QCD does not give a mass spectrum in perfect agreement with experiment so alternate ways to fix the scale would lead to different values. This is an intrinsic limitation of the quenched approximation. The hopping parameters corresponding to the strange and charm quark masses were fixed from the $\phi$-meson and D-meson respectively. These are the values given in Table II. A systematic uncertainty in these quark mass determinations was estimated by also using the kaon and $J / \psi$ masses.

The masses of hadrons containing up and down quarks have to be extrapolated into the light quark mass region. This is done by extrapolating the masses calculated at the four largest hopping parameters as a function of pion mass using the function $c_{0}+c_{2} m_{\pi}^{2}+$ $c_{3} m_{\pi}^{3}$. In some cases the mass to be extrapolated appears to be described very well by a function without an $m_{\pi}^{3}$ term. In such cases, where the coefficient $c_{3}$ comes out to be not 
statistically significant, the difference between aquadratic extrapolation and a quadratic plus cubic extrapolation is included as a contribution to the systematic error.

Some representative results of the mass determination are shown in terms of so-called effective mass plots for singly heavy baryons in Figs. 1 and 2. The effective mass $M(t)$ is $\ln (g(t) / g(t+1))$ where $g(t)$ is the zero-momentum time correlation function for the hadron. The solid line in the figures shows the ground state mass obtained by a simultaneous fit to the local and smeared correlation functions plotted over the time window of the fit, and the dashed lines indicate the bootstrap error. Note that a two exponential form was used to fit the local correlators. Corresponding results for doubly heavy baryons are given in Figs. 3 and 4.

The results for singly charmed baryons are given in Table III. The first error is the statistical error which is calculated using a bootstrap analysis employing a bootstrap sample size equal to the configuration sample size. The second error is the combined systematic error. This includes the overall scale uncertainty, the uncertainty due to choice of the correlation function fitting range as well as strange and charm quark mass uncertainties and light $(\mathrm{u}, \mathrm{d})$ quark mass extrapolation ambiguity, where applicable. For comparison, the experimental values are also shown, where they are known.

The masses of doubly charmed baryons were also calculated and are given in Table IV. No doubly heavy baryons have been observed yet in experiments but one may hope that this situation will change in the not too distant future (see Ref. [16] for a review of the possibilities for experimental observation). Note also that the spin splittings for the doubly charmed baryons are as large and perhaps even larger than in the singly charmed sector.

\section{DISCUSSION}

In order to see how our results for charmed baryons and particularly for the spin splittings fit into the overall scheme of hadron physics, it is useful to start with a slight digression and consider the spin splittings of pseudoscalar and vector mesons. It is well known that for vector $(V)$ and pseudoscalar $(P)$ meson pairs of the form $Q \bar{q}$, where $q$ is up or down and $Q$ is any light or heavy flavor, the squared mass difference $M_{V}^{2}-M_{P}^{2}$ is approximately constant for all flavors $Q$. This mass relation was first derived within the framework of string-like models for hadrons imposing the constraints of chiral symmetry 17 19. It can also be understood in the quark model with a linear confining potential 20] and, for heavy-light mesons, from heavy quark effective theory(HQET) [21]. For our purposes it is useful to factorize the squared mass difference and make a plot of the spin splitting $\Delta M_{\text {mes }}=M_{V}-M_{P}$ versus the inverse of the average meson mass $M_{\text {ave }}=\left(M_{V}+M_{P}\right) / 2$. This is given in Fig. 5 where the line is the best linear fit to the experimental points shown by triangles. The mesons pairs included in the plot are $(\rho, \pi),\left(K^{*}, K\right),\left(D^{*}, D\right)$ and $\left(B^{*}, B\right)$. The remaining points in Fig. 5 are the results from quenched lattice QCD calculations. The squares are results of the present simulation and the circles are representative results collected from a variety of published papers 
[7,9,22,23]. The underestimate of color hyperfine effects by quenched lattice QCD can be seen clearly.

We would like to have a similar global view of spin splittings in the baryon sector. In fact the string models that predict the meson squared mass difference relation give a similar relation for baryons [18]. However, if one plots the baryon mass difference $\Delta M_{b a r}=M_{3 / 2}-M_{1 / 2}$ versus the inverse of the average baryon mass $\left(M_{3 / 2}+M_{1 / 2}\right) / 2$ for the well measured baryon pairs $(\Delta, N),\left(\Sigma^{*}, \Sigma\right)$ and $\left(\Sigma_{c}^{*}, \Sigma_{c}\right)$, it is found empirically that the relation is not linear. On the other hand, if one uses the average meson mass (associating mesons and baryons with the same flavor content) an almost exact linear relation is found. This is shown in Fig. 6 where the triangles are experimental data and the line is the best linear fit. The results of the present simulation as well as some results of published quenched lattice calculations [23,24] are also shown in Fig. 6. Unfortunately, masses in the baryon sector are not determined with the same precision as for mesons but a clear qualitative difference from Fig. 5 can be seen. No suppression of the spin splitting is evident. Although not completely conclusive, there may be a tendency for baryon spin splittings to be overestimated.

The linearity of the experimental results for mass differences plotted versus a common variable in Figs. 5 and 6 implies that the ratio of mass differences should be constant. This is shown in Fig. 7 for the experimental data where the ratios of the meson to baryon mass differences $(\rho, \pi) /(\Delta, N),\left(K^{*}, K\right) /\left(\Sigma^{*}, \Sigma\right)$ and $\left(D^{*}, D\right) /\left(\Sigma_{c}^{*}, \Sigma_{c}\right)$ are plotted versus the averaged meson mass. The ratio is constant to within about $2 \%$ and the solid line is the average value 2.13. This remarkable result was anticipated by Lipkin [25] (see also Lipkin and O'Donnell [26]) within the framework of a quark model although it required a number of assumptions. For mesons and baryons with a single heavy quark, HQET also implies a constancy in the meson to baryon spin splitting but the effective theory can not predict the value of the ratio.

One would like to see what lattice QCD predicts for the meson to baryon mass difference ratio. Unfortunately the errors associated with extrapolation preclude a very precise determination. For this reason we choose to present unextrapolated results, fixing the "light" quark at a kappa value near that of the strange quark. The results for Qqq-baryons (q-mass fixed, Q-mass variable) are shown in Fig. 8. The calculated ratio is quite constant especially for the simulations at higher $\beta$ where the results are more precise. The fact that the ratio falls below the empirical value, shown by the solid line in Fig. 8, is an indication that the suppression of spin splittings is not present in baryons in the same way as it is in mesons.

For doubly heavy baryons one can make definite predictions for the relation between mesonic and baryonic spin splittings. Consider the simplest possible quark model in which only quark masses and the color hyperfine interaction term are taken into account [27,28]. The operators for meson and baryon mass are

$$
M_{\text {mes }}=m_{1}+m_{2}+a\left(\overrightarrow{\sigma_{1}} \cdot \overrightarrow{\sigma_{2}}\right) / m_{1} m_{2},
$$

and 


$$
M_{b a r}=m_{1}+m_{2}+m_{3}+\frac{1}{2} \sum_{i>j} a_{i j}^{\prime}\left(\overrightarrow{\sigma_{i}} \cdot \overrightarrow{\sigma_{j}}\right) / m_{i} m_{j} .
$$

Note the factor of $1 / 2$ in the last term of (4.2). This reflects the reduction in the strength of the gluon exchange between quarks in a color anti-triplet state relative to that between a quark and antiquark in a color singlet. Evaluating the above expressions for mesons $Q \bar{q}$ and $\Xi$ baryons $Q Q q$ one finds

$$
\begin{gathered}
M(P)=m_{q}+m_{Q}-\frac{3 c}{m_{q} m_{Q}}, \\
M(V)=m_{q}+m_{Q}+\frac{c}{m_{q} m_{Q}}, \\
M\left(\Xi_{Q Q}\right)=m_{q}+2 m_{Q}+2\left\{\frac{c_{Q Q}^{\prime}}{4 m_{Q}^{2}}-\frac{c_{q Q}^{\prime}}{m_{q} m_{Q}}\right\}, \\
M\left(\Xi_{Q Q}^{*}\right)=m_{q}+2 m_{Q}+2\left\{\frac{c_{Q Q}^{\prime}}{4 m_{Q}^{2}}+\frac{c_{q Q}^{\prime}}{2 m_{q} m_{Q}}\right\},
\end{gathered}
$$

where the coefficients $c$ and $c^{\prime}$ depend on the probability of finding the interacting quarks at zero separation. The relation between the spin splittings is

$$
\Delta M_{b a r}=\frac{3}{4} \frac{c_{q Q}^{\prime}}{c} \Delta M_{m e s}
$$

Now, if it is assumed that the two heavy quarks act as a single heavy compact antitriplet color source, it is reasonable to expect that $c_{q Q}^{\prime} \approx c$ so that

$$
\Delta M_{b a r} \approx \frac{3}{4} \Delta M_{m e s}
$$

This diquark picture for doubly heavy baryons can also be analyzed in a heavy quark effective theory. This has been done in an elegant formulation utilizing a superflavor symmetry to relate hadrons containing a heavy vector diquark to those with a heavy spin-1/2 antiquark [29].

Although no evidence yet exists that for doubly heavy baryons the ratio of meson to baryon spin splittings is a constant function of quark mass our lattice calculations suggest that it might be so. Fig. 9 shows the ratio of unextrapolated results for $Q Q q-$ baryons (q-mass fixed, Q-mass variable). The long dashed line in the figure is the value of the ratio obtained experimentally for the strange quark, that is the $K^{*}-K$ mass difference divided by the $\Xi^{*}-\Xi$ mass difference. The short dashed line is the prediction Eq. (4.8).

Eq. (4.7) has been derived explicitly for the doubly heavy $\Xi_{Q Q}$, $\Xi_{Q Q}^{*}$ system but the same expression holds for the singly heavy baryons. In this case there is no simple relation between $c_{q Q}^{\prime}$ and $c$ but, since the gluon interaction between quarks is weaker than between a quark and antiquark, one might expect that $c_{q Q}^{\prime}<c$. This implies that spin 
splittings in singly heavy baryons are smaller than in doubly heavy baryons containing the same quark flavors. This expectation is borne out empirically for the strange $\Sigma^{\prime} s$ and $\Xi^{\prime} s$ but given the statistical and systematic errors there is no clear evidence from our lattice simulations for charmed baryons.

Finally, we note that if it is assumed that the ratio $\Delta M_{m e s} / \Delta M_{b a r}$ is constant as a function of quark mass for doubly heavy baryons just as it is for singly heavy baryons, then using the experimental values of the $\Xi$ and $\Xi^{*}$ masses along with the known meson masses yields the phenomenological predictions

$$
\Xi_{c c}^{*}-\Xi_{c c}=76.6 \mathrm{MeV}
$$

and

$$
\Xi_{b b}^{*}-\Xi_{b b}=24.5 \mathrm{MeV}
$$

\section{SUMMARY}

Masses of charmed baryons were calculated in quenched lattice QCD using improved gluon and quark actions on an anisotropic lattice. The actions were improved to remove tree level errors up to $O\left(a^{2}\right)$ and tadpole factors, estimated by using gauge field links in Landau gauge, were introduced to remove the dominant errors due to tadpole fluctuations.

Calculations were done at three different values of the gauge coupling constant with the spatial lattice spacing varying from $0.22 \mathrm{fm}$ to $0.15 \mathrm{fm}$. The results at the two largest values of the gauge coupling are compatible with each other and are in fair agreement with experimental data where it is available. Masses and mass differences at the smallest value of the gauge coupling (largest lattice spacing) tend to be smaller, perhaps reflecting larger finite lattice spacing errors.

The main focus of our work was the mass differences due to spin dependent interactions. It is well established that in quenched lattice QCD the mass differences between vector and pseudoscalar mesons are underestimated for mesons containing heavy quarks. The present simulations show no comparable suppression of splittings between spin $1 / 2$ and spin $3 / 2$ baryons. Our results and results taken from the literature indicate a tendency for baryon spin splittings to be overestimated but, this is not established definitively due to relatively large errors associated with baryon mass determinations.

To get an overall view of the spin splittings it was useful to consider the scaling of mass differences with the average meson mass as one changes quark flavors. For mesons this relation 17 predates QCD. We have found it useful to extend the same scaling (with average meson mass) to mass differences between baryons. A relation between meson and baryon spin splittings, which is implied by this scaling, was anticipated by Lipkin 25 from a quark model analysis. From the point of view of QCD, a mass independent ratio of meson to baryon spin splittings can be derived for heavy flavored mesons and baryons 
using heavy quark effective theory, but how to extend this result to light flavor hadrons is not clear. Nonetheless a constant meson to baryon mass difference ratio is satisfied remarkably well by experimental data for quark flavors from light to charm. Obviously, a definitive experimental determination of the $\Sigma_{b}^{*}$ mass to extend this analysis to the b-quark region would be extremely useful.

Our lattice QCD results are compatible with a mass independent meson to baryon spin splitting ratio for both singly and doubly heavy baryons. However, the more precise values obtained at our larger values of the gauge coupling indicate a value for the ratio for singly heavy baryons smaller than the empirical value. This can be interpreted as another indication that the suppression of spin splittings found for mesons does not occur for baryons. For doubly heavy baryons our preferred values for the meson to baryon ratio lie below the value obtained by using the masses of the doubly strange hyperons $\Xi$ and $\Xi^{*}$. Clearly, the experimental observation of doubly heavy baryons and the systematic investigation of their spin splittings would be very interesting indeed.

\section{ACKNOWLEDGMENTS}

The authors are grateful to Mark Wise for a helpful communication. This research was supported in part by the Natural Sciences and Engineering Research Council of Canada. Some of the computing was done on hardware funded by the Canada Foundation for Innovation, with contributions from Compaq Canada, Avnet Enterprise Solutions, and the Government of Saskatchewan.

\section{APPENDIX A: THE LATTICE ACTION}

The lattice action has two terms: gauge action and quark action. The entire action is classically and tadpole-improved with the tadpole factors, $u_{s}$ and $u_{t}$, defined as the mean links in Landau gauge in a spatial and temporal direction, respectively.

The gauge field action is

$$
\begin{aligned}
S_{G}(U)= & \frac{5 \beta}{3}\left[\frac{1}{u_{s}^{4} \xi} \sum_{\mathrm{ps}}\left(1-\frac{1}{3} \operatorname{Re} \operatorname{Tr} U_{\mathrm{ps}}\right)-\frac{1}{20 u_{s}^{6} \xi} \sum_{\mathrm{rs}}\left(1-\frac{1}{3} \operatorname{Re} \operatorname{Tr} U_{\mathrm{rs}}\right)\right. \\
& +\frac{\xi}{u_{s}^{2} u_{t}^{2}} \sum_{\mathrm{pt}}\left(1-\frac{1}{3} \operatorname{Re} \operatorname{Tr} U_{\mathrm{pt}}\right)-\frac{\xi}{20 u_{s}^{4} u_{t}^{2}} \sum_{\mathrm{rst}}\left(1-\frac{1}{3} \operatorname{Re} \operatorname{Tr} U_{\mathrm{rst}}\right) \\
- & \left.-\frac{\xi}{20 u_{s}^{2} u_{t}^{4}} \sum_{\mathrm{rts}}\left(1-\frac{1}{3} \operatorname{Re} \operatorname{Tr} U_{\mathrm{rts}}\right)\right],
\end{aligned}
$$

where $\xi \equiv a_{s} / a_{t}$ is the aspect ratio and $\beta$ is the lattice gauge field coupling constant. The subscripts "ps" and "rs" denote spatial plaquettes and spatial planar $1 \times 2$ rectangles respectively. Plaquettes in the temporal-spatial planes are denoted by "pt", while 
rectangles with the long side in a spatial(temporal) direction are labeled by "rst" ("rts"). The leading classical errors of this action are quartic in lattice spacing.

An action of the D234 type [8] is used for the quarks with coefficients set to their tadpole-improved classical values. Its leading classical errors are cubic in lattice spacing:

$$
\begin{aligned}
S_{F}(\bar{q}, q ; U)= & \frac{4 \kappa}{3} \sum_{x, i}\left[\frac{1}{u_{s} \xi^{2}} D_{1 i}(x)-\frac{1}{8 u_{s}^{2} \xi^{2}} D_{2 i}(x)\right] \\
& +\frac{4 \kappa}{3} \sum_{x}\left[\frac{1}{u_{t}} D_{1 t}(x)-\frac{1}{8 u_{t}^{2}} D_{2 t}(x)\right] \\
& +\frac{2 \kappa}{3 u_{s}^{4} \xi^{2}} \sum_{x, i<j} \bar{\psi}(x) \sigma_{i j} F_{i j}(x) \psi(x) \\
& +\frac{2 \kappa}{3 u_{s}^{2} u_{t}^{2} \xi} \sum_{x, i} \bar{\psi}(x) \sigma_{0 i} F_{0 i}(x) \psi(x) \\
& -\sum_{x} \bar{\psi}(x) \psi(x),
\end{aligned}
$$

where $\kappa$ denotes the hopping parameter and

$$
\begin{aligned}
D_{1 i}(x)= & \bar{\psi}(x)\left(1-\xi \gamma_{i}\right) U_{i}(x) \psi(x+\hat{i}) \\
+ & \bar{\psi}(x+\hat{i})\left(1+\xi \gamma_{i}\right) U_{i}^{\dagger}(x) \psi(x), \\
D_{1 t}(x)= & \bar{\psi}(x)\left(1-\gamma_{4}\right) U_{4}(x) \psi(x+\hat{t}) \\
+ & \bar{\psi}(x+\hat{t})\left(1+\gamma_{4}\right) U_{4}^{\dagger}(x) \psi(x), \\
D_{2 i}(x)= & \bar{\psi}(x)\left(1-\xi \gamma_{i}\right) U_{i}(x) U_{i}(x+\hat{i}) \psi(x+2 \hat{i}) \\
+ & \bar{\psi}(x+2 \hat{i})\left(1+\xi \gamma_{i}\right) U_{i}^{\dagger}(x+\hat{i}) U_{i}^{\dagger}(x) \psi(x), \\
D_{2 t}(x)= & \bar{\psi}(x)\left(1-\gamma_{4}\right) U_{4}(x) U_{4}(x+\hat{t}) \psi(x+2 \hat{t}) \\
+ & \bar{\psi}(x+2 \hat{t})\left(1+\gamma_{4}\right) U_{4}^{\dagger}(x+\hat{t}) U_{4}^{\dagger}(x) \psi(x), \\
g F_{\mu \nu}(x)= & \frac{1}{2 i}\left(\Omega_{\mu \nu}(x)-\Omega_{\mu \nu}^{\dagger}(x)\right)-\frac{1}{3} \operatorname{Im}\left(\operatorname{Tr} \Omega_{\mu \nu}(x)\right), \\
\Omega_{\mu \nu}= & \frac{-1}{4}\left[U_{\mu}(x) U_{\nu}(x+\hat{\mu}) U_{\mu}^{\dagger}(x+\hat{\nu}) U_{\nu}^{\dagger}(x)\right. \\
& +U_{\nu}(x) U_{\mu}^{\dagger}(x-\hat{\mu}+\hat{\nu}) U_{\nu}^{\dagger}(x-\hat{\mu}) U_{\mu}(x-\hat{\mu}) \\
& +U_{\mu}^{\dagger}(x-\hat{\mu}) U_{\nu}^{\dagger}(x-\hat{\mu}-\hat{\nu}) U_{\mu}(x-\hat{\mu}-\hat{\nu}) U_{\nu}(x-\hat{\nu}) \\
& \left.+U_{\nu}^{\dagger}(x-\hat{\nu}) U_{\mu}(x-\hat{\nu}) U_{\nu}(x+\hat{\mu}-\hat{\nu}) U_{\mu}^{\dagger}(x)\right] .
\end{aligned}
$$




\section{REFERENCES}

[1] For a review see C. Bernard, Nucl. Phys. B(Proc. Suppl.) 94, 159(2001).

[2] C. Stewart and R. Koniuk, Phys. Rev. D 63, 054503(2001).

[3] CP-PACS Collaboration, T. Manke et al., Phys. Rev. D 62, 114508(2000).

[4] S. Collins et al., Phys. Rev. D 60, 074504(1999).

[5] UKQCD Collaboration, K.C. Bowler et al., Phys. Rev. D 54, 3619(1996).

[6] R.M. Woloshyn, Phys. Lett. B 476, 309(2000).

[7] A. Ali Khan et al., Phys. Rev. D 62, 054505(2001).

[8] M. Alford, T.R. Klassen, and G.P. Lepage, Nucl.Phys. B496, 377(1997).

[9] R. Lewis and R.M. Woloshyn, Phys. Rev. D 62, 114507(2000).

[10] D.B. Leinweber, R.M. Woloshyn, and T. Draper, Phys. Rev. D 43, 1659(1991).

[11] R.M. Woloshyn, Nucl. Phys. B(Proc. Suppl). 93, 38(2001).

[12] M. Benmerrouche, R.M. Davidson, and N.C. Mukhopadhyay, Phys. Rev. C 39, 2339(1989).

[13] C. Alexandrou, S. Güsken, F. Jegerlehner, K, Schilling, and R. Sommer, Nucl. Phys. B414, 815(1994).

[14] D.E. Groom et al., Eur. Phys. J. C 15, 1(2000).

[15] CLEO Collaboration, C.P. Jessop et al., Phys. Rev. Lett. 82, 492(1999).

[16] V.V Kiselev and A.K. Likhoded, hep-ph/0103169.

[17] M. Ademollo, G. Veneziano, and S. Weinberg, Phys. Rev. Lett. 22, 83(1969).

[18] D.C. Lewellen, Nucl. Phys. B392, 137(1992).

[19] S.R. Beane, Phys. Rev. D 59, 036001(1999).

[20] M. Frank and P.J. O'Donnell, Phys. Lett. B 159, 174(1985).

[21] See. for example, M.B. Wise, hep-ph/9805468.

[22] J. Hein et al., Phys. Rev. D 62, 074503(2000); K.I. Ishikawa et al., Phys. Rev. D 61, 074501(2000); P. Boyle, Nucl. Phys. B(Proc. Suppl.) 63A-C, 314(1998).

[23] CP-PACS Collaboration, S. Aoki et al., Phys. Rev. Lett. 84, 238(2000); UKQCD Collaboration, K.C. Bowler et al., Phys. Rev. D 63, 054506(2000); F. Butler et al., Nucl. Phys. B 430, 179(1994).

[24] T. Bhattacharya, R. Gupta, G. Kilcup, and S. Sharpe, Phys. Rev. D 53, 6486(1996).

[25] H.J. Lipkin, Phys. Lett. B 171, 293(1986).

[26] H.J. Lipkin and P.J. O’Donnell, Phys. Lett. B 409, 412(1997).

[27] F. Halzen and A.D. Martin, Quarks 83 Leptons (Wiley, New York, 1984)Chap. 2.

[28] J.F. Donoghue, E. Golowich, and B.R. Holstein, Dynamics of the Standard Model (Cambridge University Press, Cambridge, 1992)Chap. 11.

[29] M.J. Savage and M.B. Wise, Phys. Lett. B 248, 177(1990). 


\section{TABLES}

TABLE I. Parameters for lattices used in this work.

\begin{tabular}{cccccc}
\hline \hline$\beta$ & size & configurations & $a_{t}^{-1}(\mathrm{GeV})$ & $u_{s}$ & $u_{t}$ \\
\hline 2.1 & $12^{3} \times 32$ & 720 & $1.803(42)$ & 0.7858 & 0.9472 \\
2.3 & $14^{3} \times 38$ & 442 & $2.210(72)$ & 0.8040 & 0.9525 \\
2.5 & $18^{3} \times 46$ & 325 & $2.625(67)$ & 0.8185 & 0.9564 \\
\hline \hline
\end{tabular}

TABLE II. Hopping parameter values. The quantities $\kappa_{s}$ and $\kappa_{c}$ are the hopping parameter values associated with strange and charm quarks respectively.

\begin{tabular}{cccc}
\hline \hline$\beta$ & $\kappa^{\prime} s$ & $\kappa_{s}(\phi)$ & $\kappa_{c}(D)$ \\
\hline 2.1 & $0.175,0.176,0.229,0.233,0.237,0.240$ & 0.2338 & 0.1739 \\
2.3 & $0.183 .0 .189,0.229,0.233,0.237,0.240$ & 0.2371 & 0.1875 \\
2.5 & $0.193,0.197,0.230,0.234,0.238,0.240$ & 0.2382 & 0.1964 \\
\hline \hline
\end{tabular}

TABLE III. Masses of singly charmed baryons. Masses are given in $\mathrm{GeV}$, mass differences are in $\mathrm{MeV}$. The first error is the statistical error and the second is the combined systematic error. The experimental values are taken from [14] except for $\Xi_{c}^{\prime}$ which is from [15].

\begin{tabular}{ccccc}
\hline \hline & $\beta=2.1$ & $\beta=2.3$ & $\beta=2.5$ & Experiment \\
\hline$\Lambda_{c}$ & $2.272(32)\left(\begin{array}{l}15 \\
23\end{array}\right)$ & $2.295(11)\left(\begin{array}{l}11 \\
15\end{array}\right)$ & $2.333(20))\left(\begin{array}{l}10 \\
10\end{array}\right)$ & 2.285 \\
$\Sigma_{c}$ & $2.379(31)\left(\begin{array}{l}23 \\
18\end{array}\right)$ & $2.490(14)\left(\begin{array}{l}17 \\
33\end{array}\right)$ & $2.493(22)\left(\begin{array}{l}21 \\
29\end{array}\right)$ & 2.455 \\
$\Sigma_{c}^{*}$ & $2.440(36)\left(\begin{array}{l}18 \\
31\end{array}\right)$ & $2.572(16)\left(\begin{array}{l}23 \\
36\end{array}\right)$ & $2.569(26)\left(\begin{array}{l}23 \\
29\end{array}\right)$ & 2.519 \\
$\Xi_{c}$ & $2.455(17)\left(\begin{array}{l}11 \\
42\end{array}\right)$ & $2.462(14)\left(\begin{array}{c}5 \\
30\end{array}\right)$ & $2.481(14)\left(\begin{array}{l}1 \\
34\end{array}\right)$ & 2.468 \\
$\Xi_{c}^{\prime}$ & $2.531(17)\left(\begin{array}{l}11 \\
35\end{array}\right)$ & $2.594(12)\left(\begin{array}{l}6 \\
25\end{array}\right)$ & $2.604(13)\left(\begin{array}{l}8 \\
30\end{array}\right)$ & 2.560 \\
$\Xi_{c}^{*}$ & $2.583(20)\left(\begin{array}{l}16 \\
40\end{array}\right)$ & $2.675(15)\left(\begin{array}{l}12 \\
29\end{array}\right)$ & $2.682(15)\left(\begin{array}{l}13 \\
28\end{array}\right)$ & 2.645 \\
$\Omega_{c}$ & $2.671(11)\left(\begin{array}{l}11 \\
59\end{array}\right)$ & $2.699(10)\left(\begin{array}{l}8 \\
41\end{array}\right)$ & $2.700(11)\left(\begin{array}{l}8 \\
40\end{array}\right)$ & 2.704 \\
$\Omega_{c}^{*}$ & $2.722(12)\left(\begin{array}{l}16 \\
58\end{array}\right)$ & $2.772(12)\left(\begin{array}{l}3 \\
43\end{array}\right)$ & $2.769(12)\left(\begin{array}{l}3 \\
40\end{array}\right)$ & \\
$\Sigma_{c}^{*}-\Sigma_{c}$ & $62(33)\left(\begin{array}{l}19 \\
32\end{array}\right)$ & $82(12)\left(\begin{array}{l}9 \\
6\end{array}\right)$ & $76(19)\left(\begin{array}{l}15 \\
4\end{array}\right)$ & 64 \\
$\Xi_{c}^{*}-\Xi_{c}^{\prime}$ & $52(15)\left(\begin{array}{l}8 \\
4\end{array}\right)$ & $82(10)\left(\begin{array}{l}8 \\
5\end{array}\right)$ & $77(9)\left(\begin{array}{l}7 \\
5\end{array}\right)$ & 70 \\
$\Omega_{c}^{*}-\Omega_{c}$ & $50(17)\left(\begin{array}{c}11 \\
6\end{array}\right)$ & $73(8)\left(\begin{array}{l}7 \\
5\end{array}\right)$ & $69(7)\left(\begin{array}{c}5 \\
6\end{array}\right)$ & \\
\hline \hline
\end{tabular}


TABLE IV. Masses of doubly charmed baryons. Masses are given in $\mathrm{GeV}$, mass differences are in $\mathrm{MeV}$. The first error is the statistical error and the second is the combined systematic error.

\begin{tabular}{cccc}
\hline \hline & $\beta=2.1$ & $\beta=2.3$ & $\beta=2.5$ \\
\hline$\Xi_{c c}$ & $3.608(15)\left(\begin{array}{c}13 \\
35\end{array}\right)$ & $3.595(12)\left(\begin{array}{l}21 \\
22\end{array}\right)$ & $3.605(12)\left(\begin{array}{l}23 \\
19\end{array}\right)$ \\
$\Xi_{c c}^{*}$ & $3.666(18)\left(\begin{array}{l}18 \\
34\end{array}\right)$ & $3.678(15)\left(\begin{array}{l}18 \\
23\end{array}\right)$ & $3.685(14)\left(\begin{array}{l}19 \\
17\end{array}\right)$ \\
$\Omega_{c c}$ & $3.747(9)\left(\begin{array}{l}11 \\
47\end{array}\right)$ & $3.727(9)\left(\begin{array}{c}16 \\
40\end{array}\right)$ & $3.733(9)\left(\begin{array}{c}7 \\
38\end{array}\right)$ \\
$\Omega_{c c}^{*}$ & $3.804(13)\left(\begin{array}{l}18 \\
54\end{array}\right)$ & $3.800(11)\left(\begin{array}{l}10 \\
36\end{array}\right)$ & $3.801(9)\left(\begin{array}{l}3 \\
34\end{array}\right)$ \\
$\Xi_{c c}^{*}-\Xi_{c c}$ & $58(14)\left(\begin{array}{c}16 \\
10\end{array}\right)$ & $83(8)\left(\begin{array}{c}7 \\
10\end{array}\right)$ & $80(10)\left(\begin{array}{l}3 \\
7\end{array}\right)$ \\
$\Omega_{c c}^{*}-\Omega_{c c}$ & $57(8)\left(\begin{array}{c}10 \\
9\end{array}\right)$ & $72(5)\left(\begin{array}{l}4 \\
5\end{array}\right)$ & $68(5)\left(\begin{array}{l}6 \\
5\end{array}\right)$ \\
\hline \hline
\end{tabular}




\section{Figure Captions}

Fig. 1 Effective mass $M(t)$ versus $t$ for spin $1 / 2$ baryons Qqq with (a) $\kappa_{q}=0.233, \kappa_{Q}=$ 0.183 (b) $\kappa_{q}=0.233, \kappa_{Q}=0.189$ (c) $\kappa_{q}=0.237, \kappa_{Q}=0.183$ (d) $\kappa_{q}=0.237, \kappa_{Q}=0.189$. The solid line indicates the ground state mass and the dashed lines the statistical error.

Fig.2 Effective mass $M(t)$ versus $t$ for spin 3/2 baryons Qqq with (a) $\kappa_{q}=0.233, \kappa_{Q}=$ 0.183 (b) $\kappa_{q}=0.233, \kappa_{Q}=0.189$ (c) $\kappa_{q}=0.237, \kappa_{Q}=0.183$ (d) $\kappa_{q}=0.237, \kappa_{Q}=0.189$. The solid line indicates the ground state mass and the dashed lines the statistical error.

Fig.3 Effective mass $M(t)$ versus $t$ for spin 1/2 baryons QQq with (a) $\kappa_{q}=0.233, \kappa_{Q}=$ 0.183 (b) $\kappa_{q}=0.233, \kappa_{Q}=0.189$ (c) $\kappa_{q}=0.237, \kappa_{Q}=0.183$ (d) $\kappa_{q}=0.237, \kappa_{Q}=0.189$. The solid line indicates the ground state mass and the dashed lines the statistical error.

Fig.4 Effective mass $M(t)$ versus $t$ for spin 3/2 baryons QQq with (a) $\kappa_{q}=0.233, \kappa_{Q}=$ 0.183 (b) $\kappa_{q}=0.233, \kappa_{Q}=0.189$ (c) $\kappa_{q}=0.237, \kappa_{Q}=0.183$ (d) $\kappa_{q}=0.237, \kappa_{Q}=0.189$. The solid line indicates the ground state mass and the dashed lines the statistical error.

Fig. 5 The mass difference between vector and pseudoscalar meson pairs plotted versus the inverse of the average mass. Triangles are experimental values, circles are results of quenched lattice calculations taken from the literature [7, 9, 22, 23] and squares the results from the present work. The line is the best linear fit to the experimental points.

Fig. 6 The mass difference between spin $1 / 2$ and spin $3 / 2$ baryon pairs plotted versus the inverse of the average mass of vector and pseudoscalar mesons with the same flavor. Triangles are experimental values, circles are results of quenched lattice calculations taken from the literature [23,24] and squares the results from the present work. The line is the best linear fit to the experimental points.

Fig. 7 The ratio of the mass difference between vector and pseudoscalar meson to the mass difference between spin $1 / 2$ and spin $3 / 2$ baryon for hadrons with the same flavor content plotted versus average meson mass. The triangles are experimental data for $(\rho, \pi) /(\Delta, N),\left(K^{*}, K\right) /\left(\Sigma^{*}, \Sigma\right)$ and $\left(D^{*}, D\right) /\left(\Sigma_{c}^{*}, \Sigma_{c}\right)$. The line is the average value.

Fig. 8 Lattice simulation results for the ratio of the mass difference between vector and pseudoscalar meson to the mass difference between singly heavy spin $1 / 2$ and spin $3 / 2$ baryon at $\beta=2.1$ (triangles), $\beta=2.3$ (circles) and $\beta=2.5$ (squares). The line is the average experimental value from Fig. 7 .

Fig. 9 Lattice simulation results for the ratio of the mass difference between vector and pseudoscalar meson to the mass difference between doubly heavy spin $1 / 2$ and spin $3 / 2$ baryon at $\beta=2.1$ (triangles), $\beta=2.3$ (circles) and $\beta=2.5$ (squares). The long dashed line is the experimental value for $\left(K^{*}, K\right) /\left(\Xi^{*}, \Xi\right)$. The short dashed line is the prediction from Eq. (4.8). 

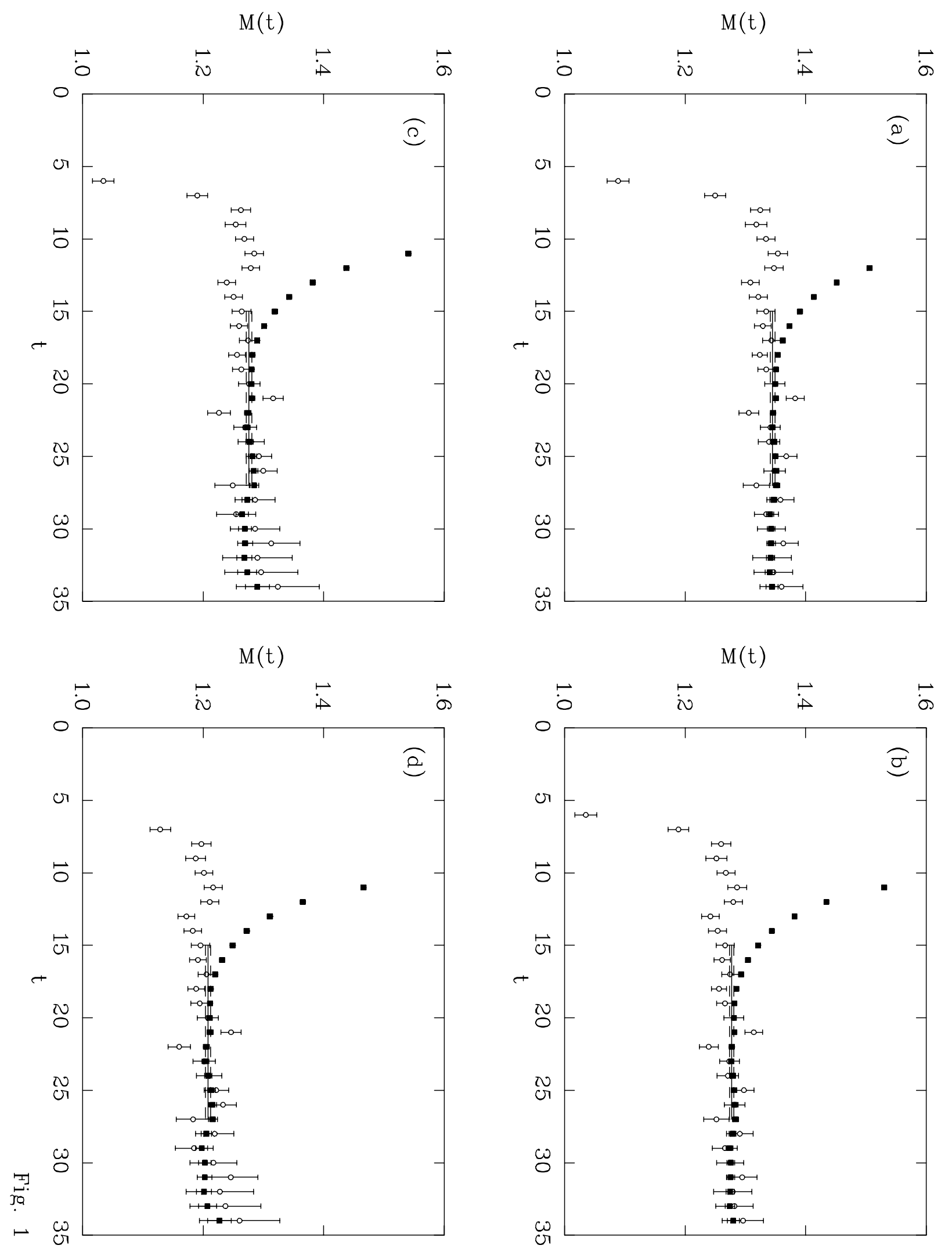

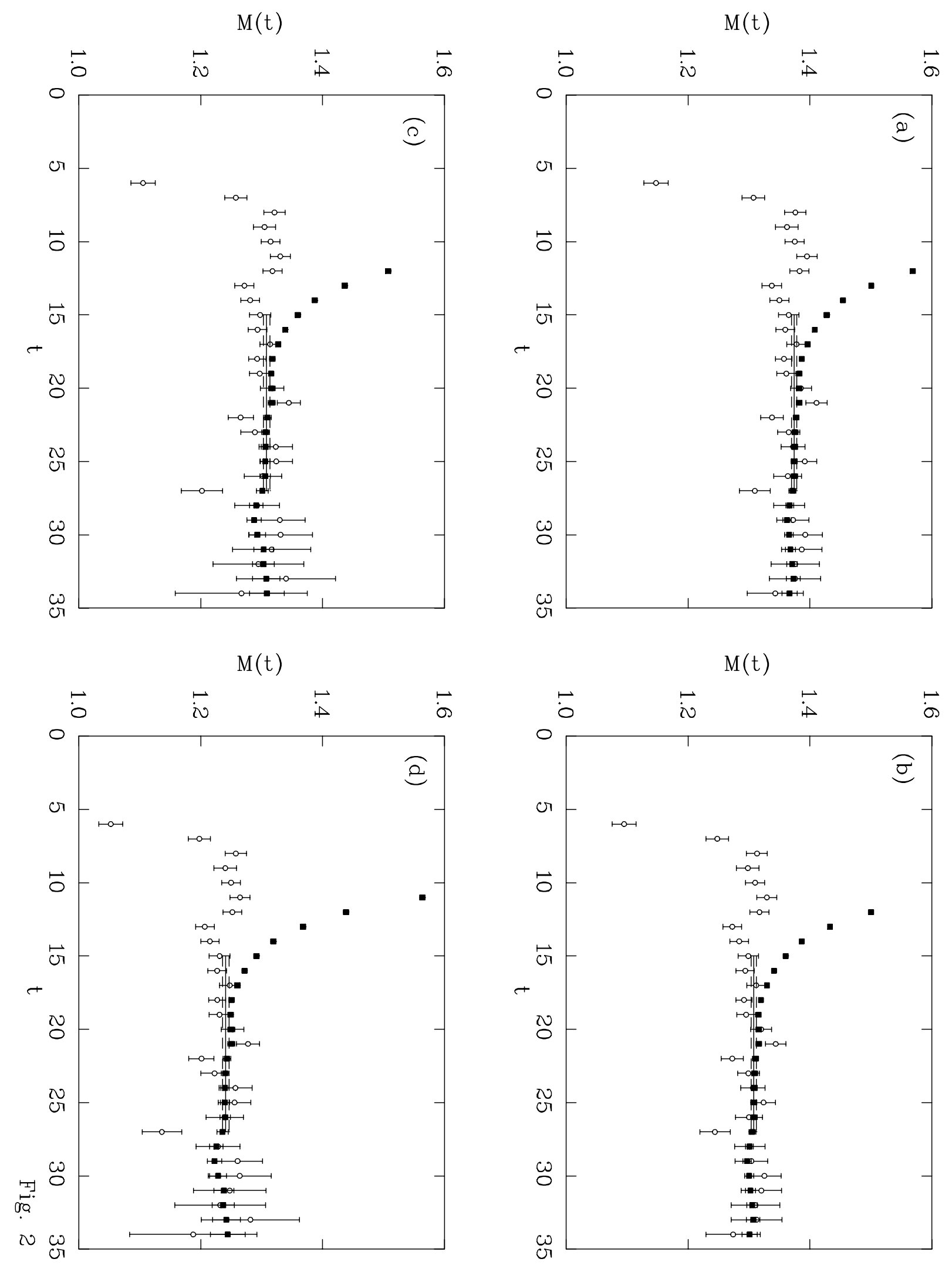

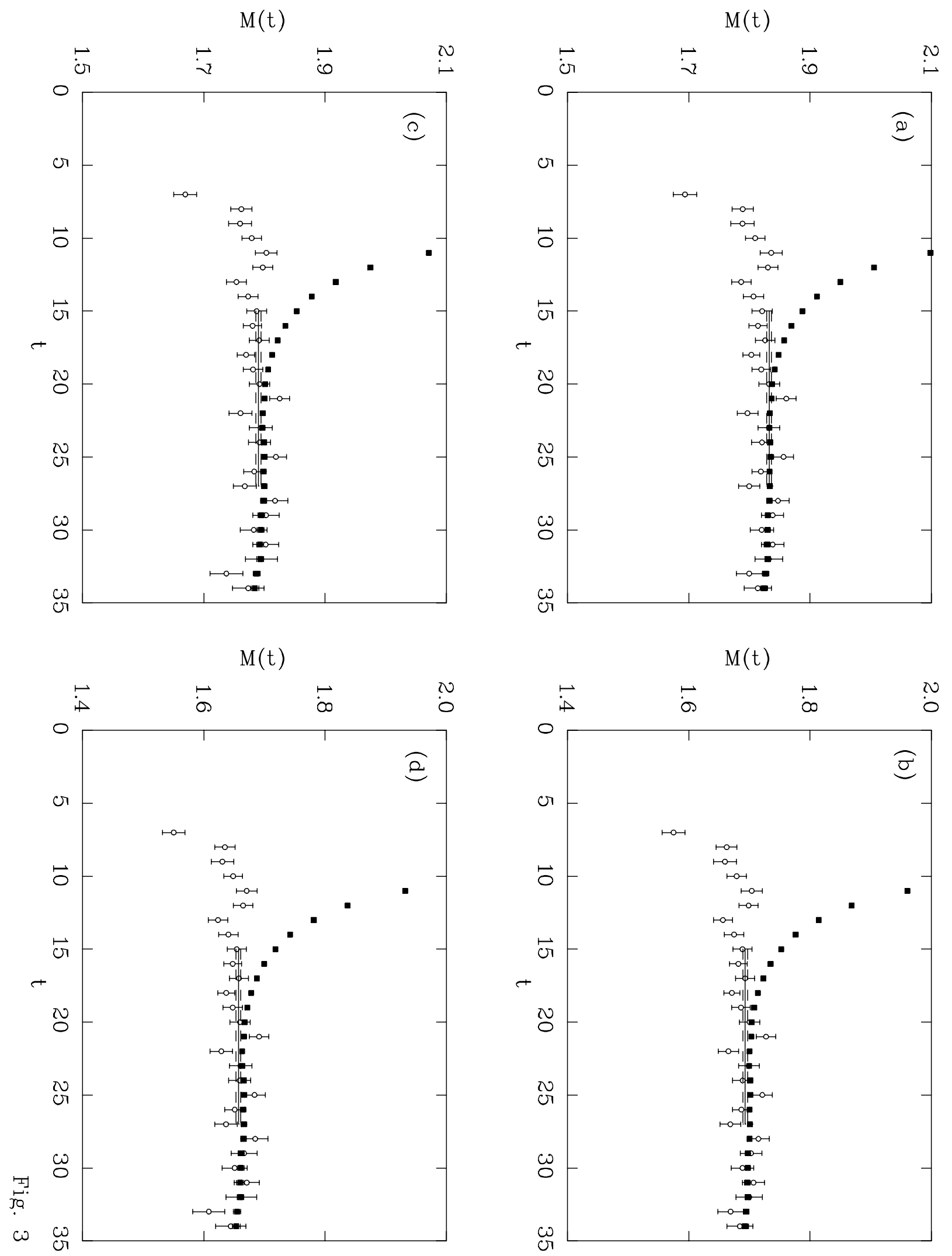

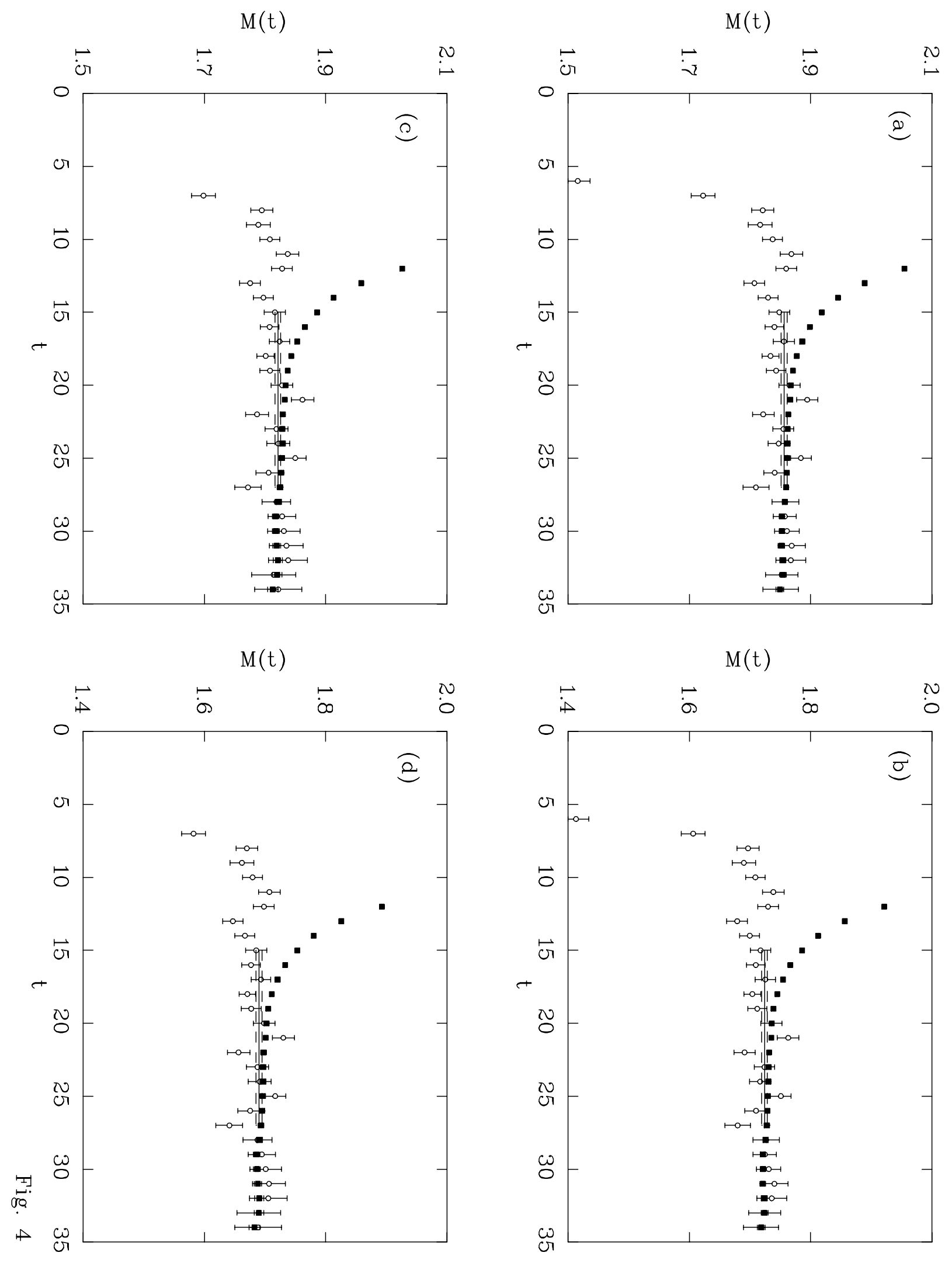


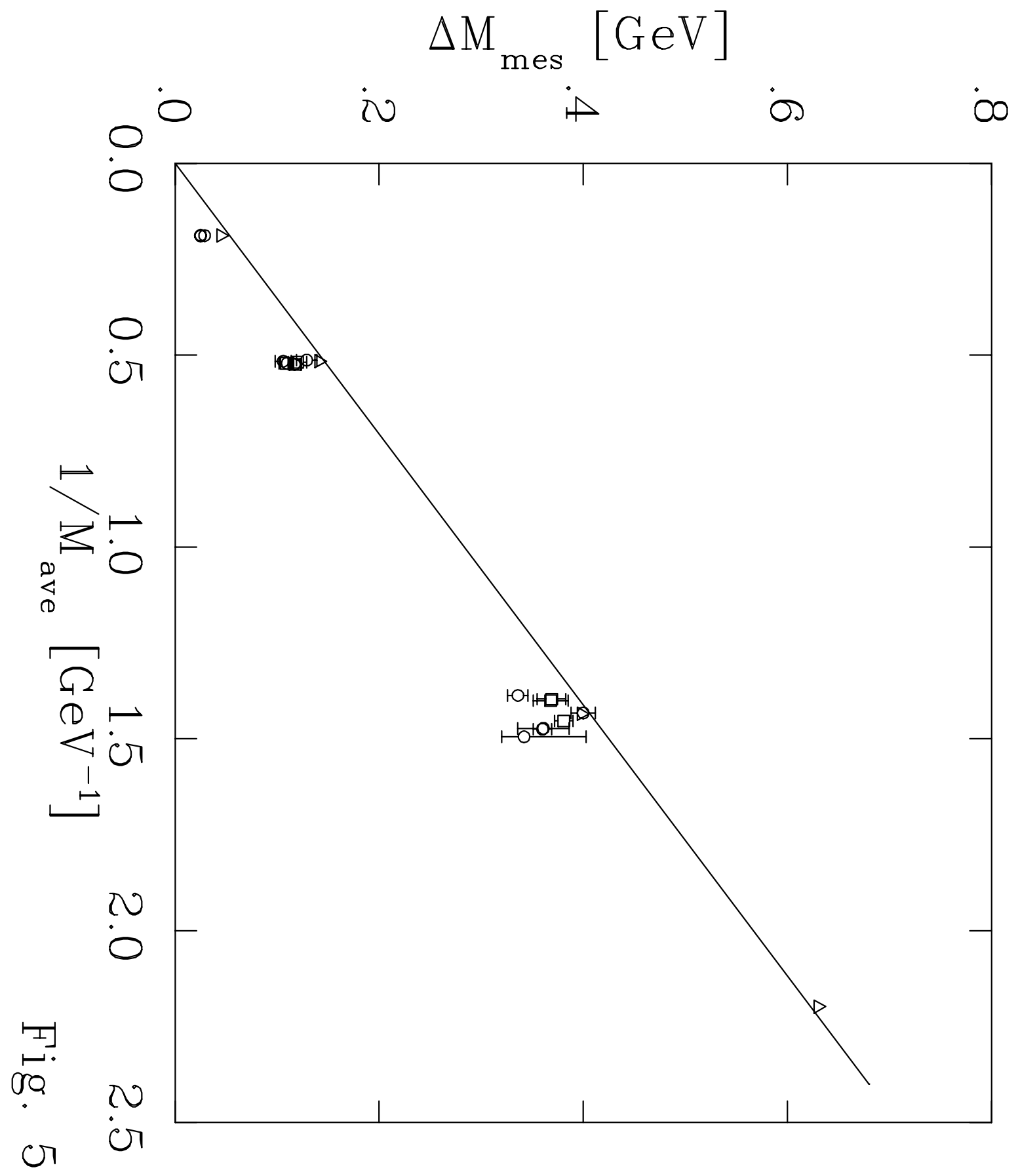




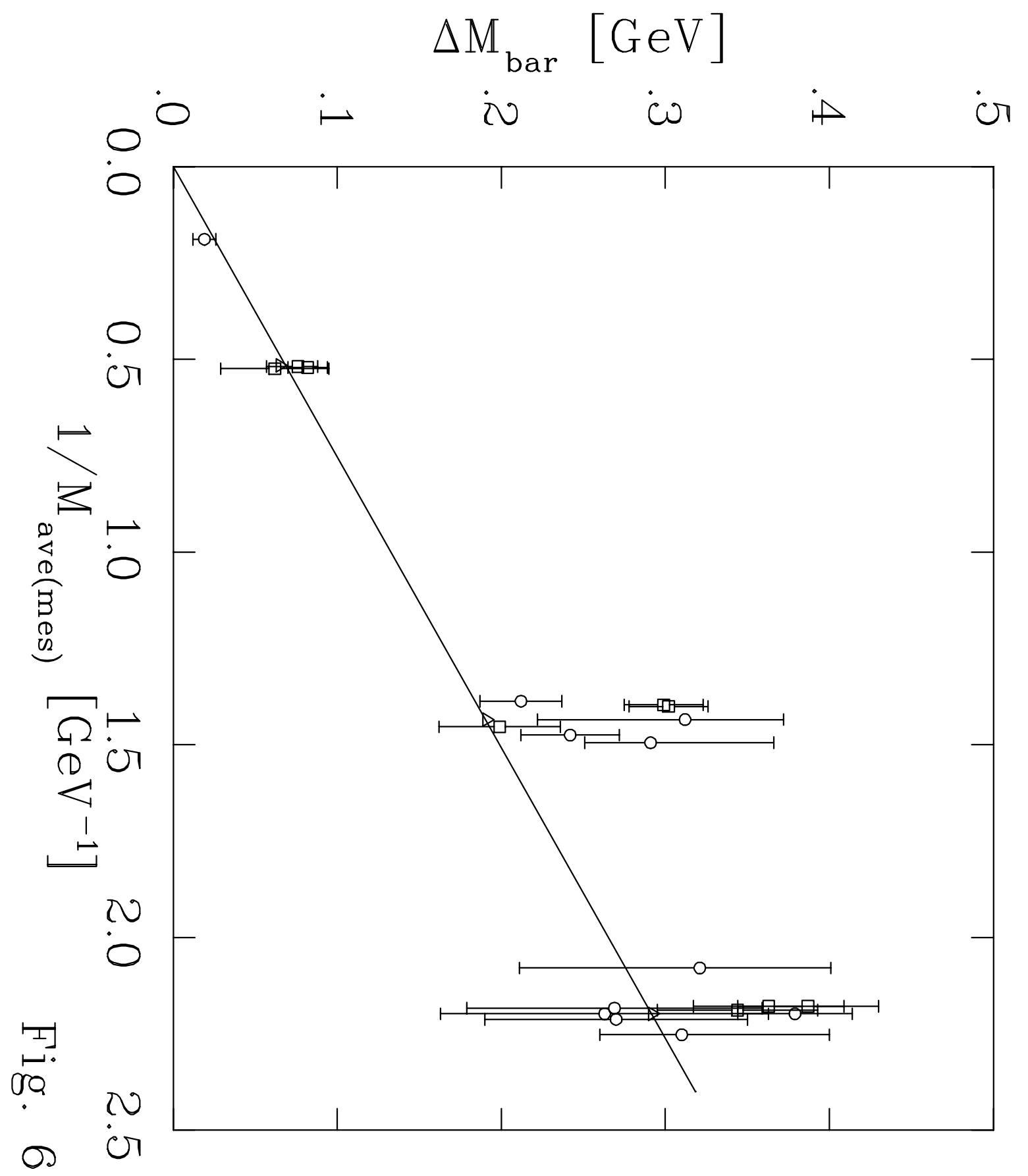




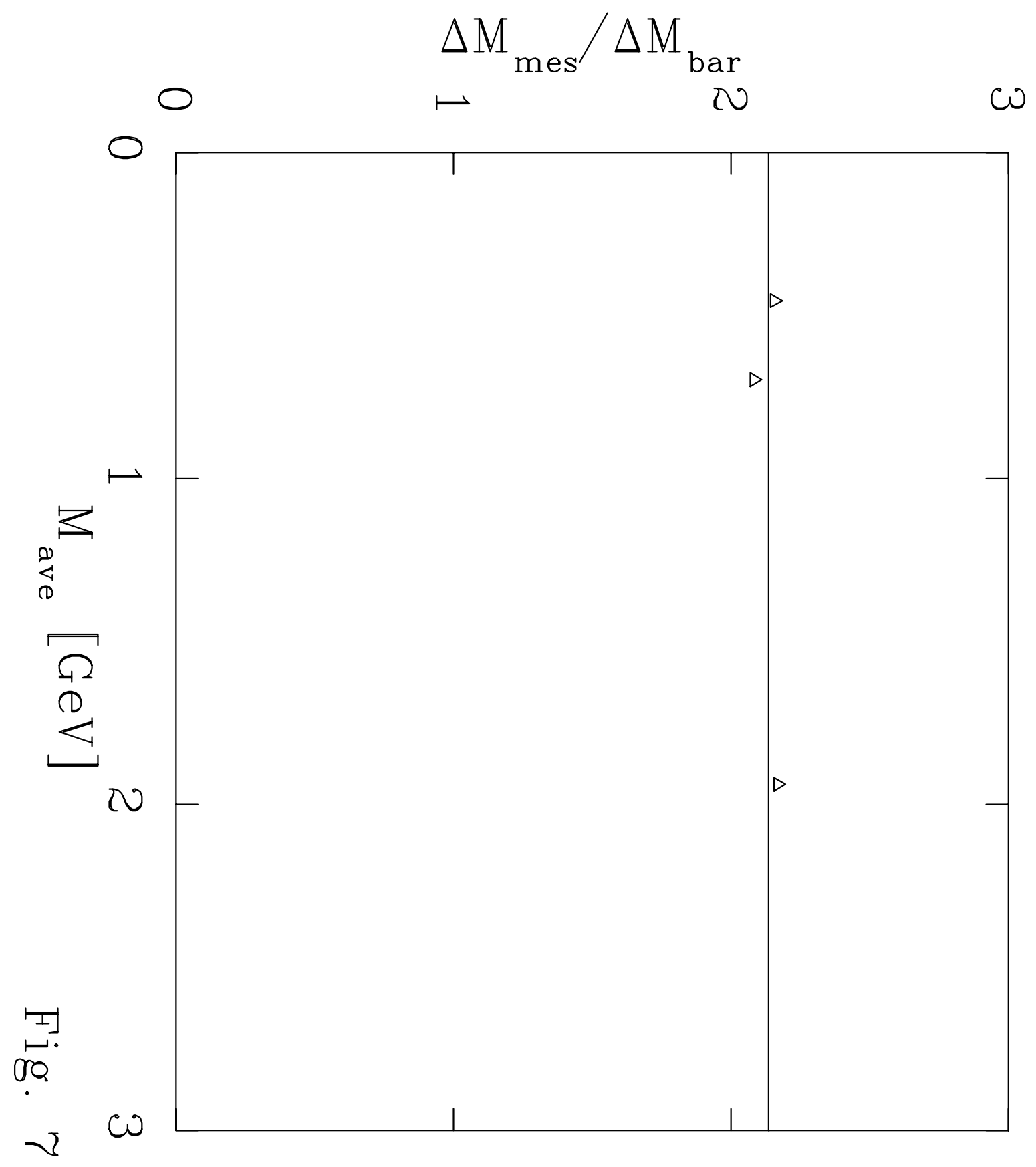




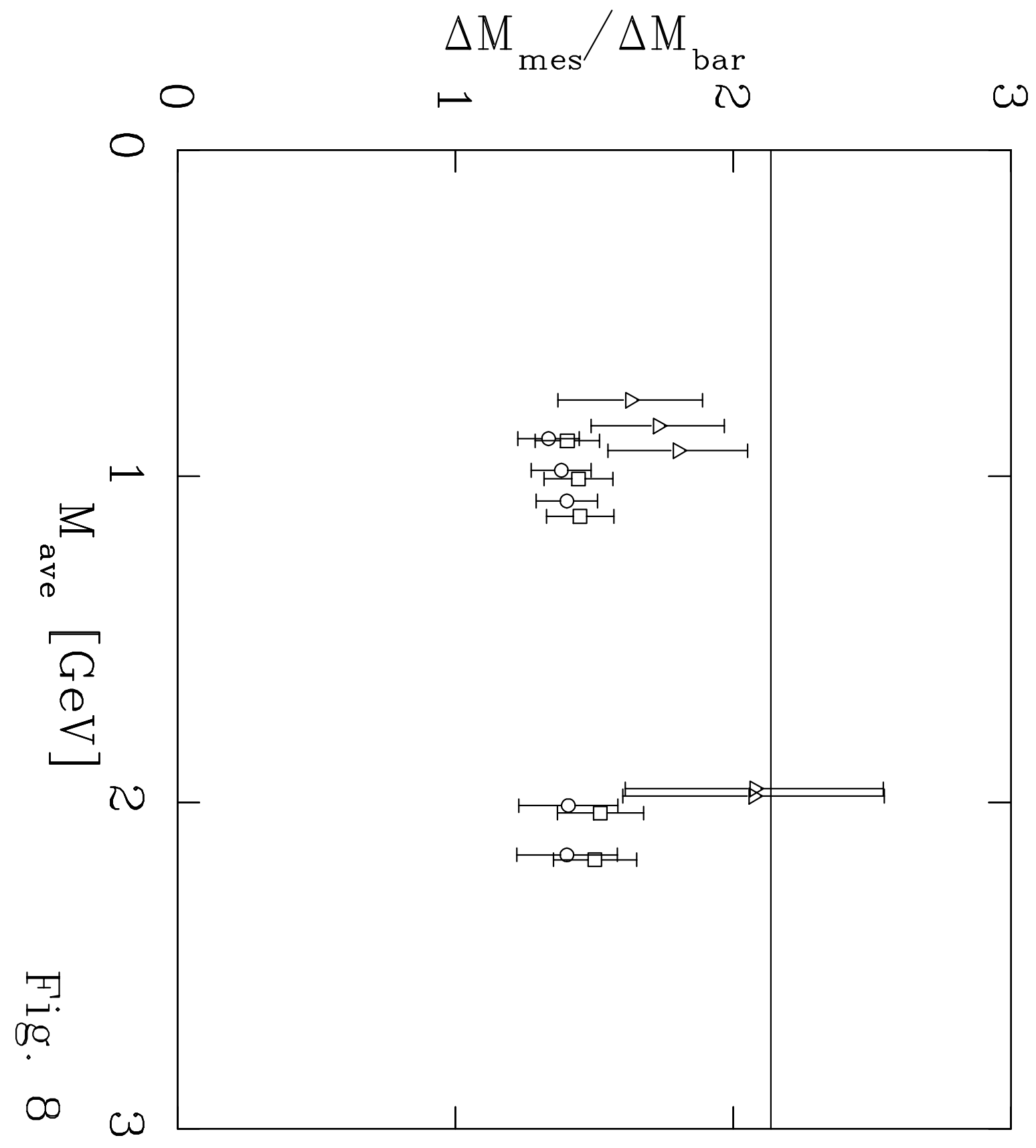




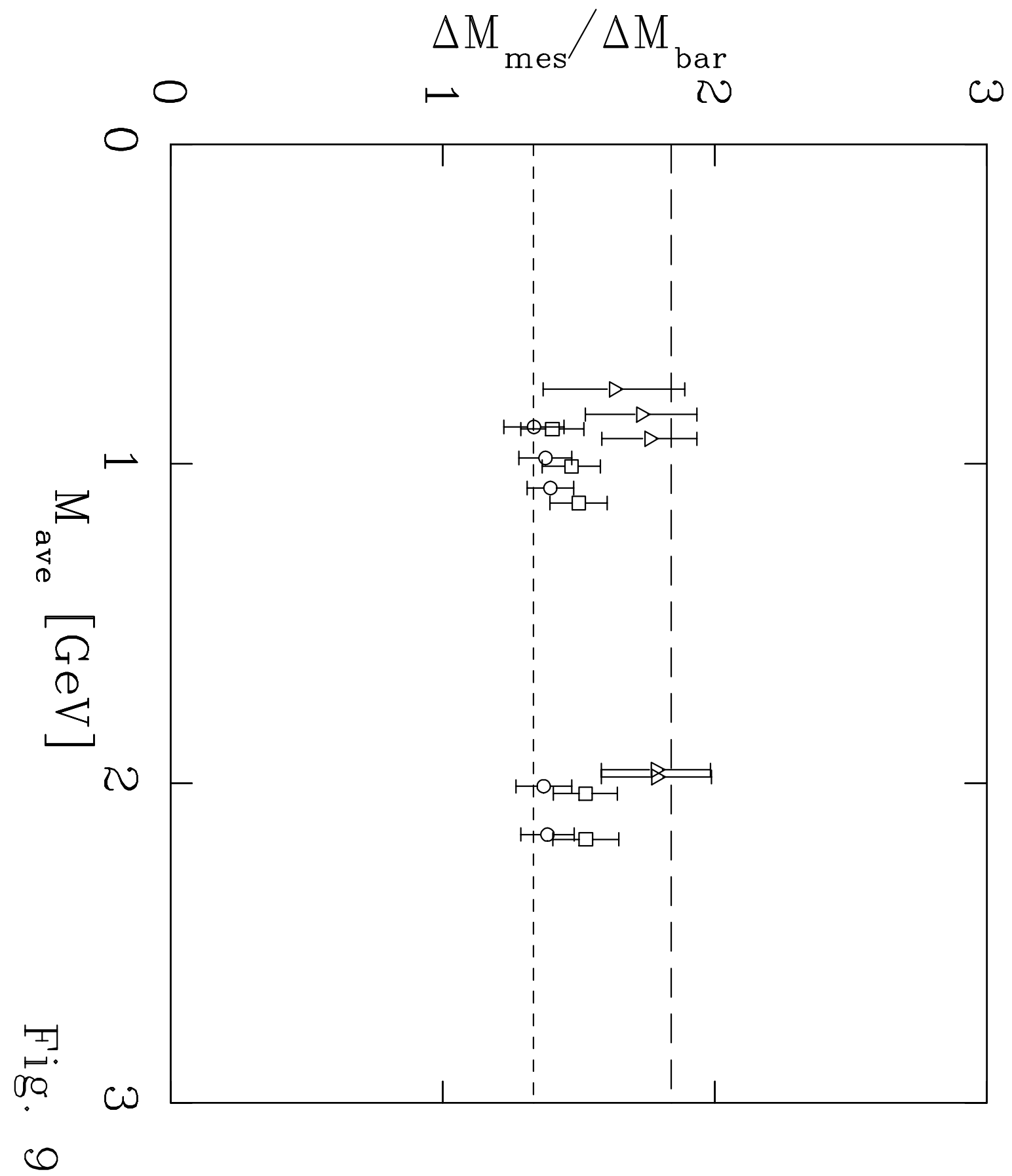

